\title{
Sources of variation in the morphological characteristics of sperm subpopulations assessed objectively by a novel automated sperm morphology analysis system
}

\author{
L. M. Thurston ${ }^{1}$, P. F. Watson ${ }^{2}$ and W. V. Holt ${ }^{1}$ \\ ${ }^{1}$ Institute of Zoology, Regent's Park, London, NW1 4RY UK; and '2Royal Veterinary College, Camden, London NW1 OTU, UK
}

\begin{abstract}
There is evidence that the mammalian ejaculate contains distinct subpopulations of spermatozoa and that the variability among these subpopulations may have adaptive and functional significance. This study investigated the precision, reproducibility and operating characteristics of a novel automated sperm morphology analysis system, the Hobson Morphology package, establishing protocols to investigate boar sperm characteristics. Five ejaculates were collected from each of three boars from different genetic lines: Landrace-Meishan introgression, Sireline Large White and Damline Large White. Five semen smears per ejaculate were stained with haematoxylin and eosin. Two hundred spermatozoa per slide were analysed. No significant differences among slides within an ejaculate were detected for sperm tail length $(P=0.770)$, head width $(P=0.736)$ and head length $(P=0.615)$, indicating that both staining and morphology analysis were precise and reproducible. Among the boars, variability in tail length was detected $(P=0.001)$, but head width $(P=0.114)$ and length $(P=0.069)$ did not differ significantly. Multivariate pattern analysis (PATN computer package) highlighted three sub-populations of spermatozoa objectively on the basis of tail length $(10.0-22.0 \mu \mathrm{m}, 22.1-73.0 \mu \mathrm{m}$ and 73.1-130.0 $\mu \mathrm{m})$. The Landrace-Meishan introgression boar possessed more spermatozoa $(P<0.0001)$ with tails $73.1-130 \mu \mathrm{m}$ long. Subsequent analysis of morphology parameters in a pure-bred Meishan boar showed similar measurements for tail length (mean $\pm \mathrm{SD} ; 66.36 \pm 24.70 \mu \mathrm{m}$ ) to the Landrace-Meishan introgression boar (mean $\pm \mathrm{SD} ; 67.09 \pm 21.80 \mu \mathrm{m}$ ). Sperm subpopulations originate during spermatogenesis, when heterogeneous genotypic effects determine the structural features of spermatozoa. The findings of this study confirm that tail length differs between boars and that subpopulations of spermatozoa can be detected within a single ejaculate.
\end{abstract}

\section{Introduction}

As technologies for studying the characteristics and functions of individual spermatozoa within an ejaculate have become more sophisticated, it has become clear that considerable heterogeneity exists. Automated analysis has been used to detect subtle morphological differences between individuals (Jagoe et al., 1987; Davis and Gravance, 1994) and it is likely that a number of distinct subpopulations of spermatozoa are present within the mammalian ejaculate (Chang and Hunter, 1975; Bedford, 1983). However, their origins and respective functions remain unclear. Variability among subgroups may be related to adaptive and functional differences within the ejaculate, possibly providing some sperm subpopulations with a selective fertility advantage (Olds-Clarke and Wivell, 1992).

It is reasonable to assume that sperm subpopulations

Received 10 December 1998 originate as a result of variations in structural formation during spermiogenesis, when heterogeneous genotypic effects can contribute to the eventual sperm structure. This hypothesis is reinforced by work on the $t$ haplotype in mice in which specific mutations cause impaired motility in a subpopulation of spermatozoa (Olds-Clarke and Sego, 1992). In addition, the process of sperm formation in a subpopulation of cells is affected during spermiogenesis, as is demonstrated by the relative increase in the proportion of morphologically abnormal spermatozoa in animals with increased inbreeding coefficients (Roldan et al., 1998).

The various attributes of sperm subpopulations have been quantified by flow cytometry (Harrison, 1996) and computerized motion analysis systems (Abaigar et al., 1999). Currently, there is little evidence to indicate that the subpopulations displaying different physiological attributes also differ morphologically (Katz and Yanagimachi, 1980). However, it may be possible to classify subpopulations on the basis of morphology using objective automated sperm morphology analysis systems (ASMA). Ultimately, a 
coupling of automated morphology analysis with a method for defining sperm physiology (for example, fluorescentactivated cell sorting, FACS) should provide a comprehensive assessment and indication of the function of sperm subpopulations.

The initial purpose of the present study was to investigate the morphological characteristics of boar spermatozoa in vitro using ASMA. A further aim was to examine the accuracy, reproducibility and operating characteristics of a novel ASMA system, the Hobson Morphology (HM) package. No information was available on the application of the Hobson package to boar spermatozoa, so it was necessary to optimize the measurement conditions for this species.

The sources of variation in sperm morphology assessment were examined to evaluate the influence of artefacts. Variation among boars and within ejaculates was explored to classify different morphological characteristics and determine the presence of sperm subgroups in individual animals. Where variation in morphology measurements among boars was observed, it was further explored by multivariate analysis to identify the presence of sperm subpopulations.

\section{Materials and Methods}

Morphometric measurements were collected to determine whether the HM offers a reliable method of assessing spermatozoa. An indication of the normality-abnormality of spermatozoa was not undertaken in this study, as a definition of 'normal' morphology introduces subjectivity into an objective protocol and excludes populations of spermatozoa falling outside 'normal' criteria. For this reason, the HM diagnostic learning module, which allows the system to be trained to make sperm classifications, was not used. Five ejaculates were collected from each of three boars and five smears were made from each ejaculate. Two hundred spermatozoa were evaluated on each slide, giving a total of 15000 spermatozoa assessed by the morphology package in this study (three boars, 15 ejaculates, 75 slides).

\section{Semen collection, processing and staining}

Ejaculates were collected each week for 5 weeks, from each of three boars from different genetic lines (Damline Large White, Sireline Large White and a Meishan-Landrace introgression backcross; Pig Improvement Company, Abingdon) using a manual collection method (King and Macpherson, 1973). Only the sperm-rich fraction of the ejaculate was collected. All boars were $1.0-1.5$ years of age, known to be fertile and were undergoing regular semen collection for commercial artificial inseminations. Sperm concentration was determined by colorimetry.

After collection, semen was diluted into a washing buffer (447 mOsmol kg-1; Cat:ZS993, IMV, L'Aigle) to a final concentration of $100 \times 10^{6} \mathrm{ml}^{-1}$ at $37^{\circ} \mathrm{C}$ and allowed to cool to room temperature at approximately $0.2^{\circ} \mathrm{C} \mathrm{min}^{-1}$. This dilution stage is the initial step in a standardized cryopreservation protocol. Five smears of spermatozoa for each ejaculate were prepared by placing $20 \mu \mathrm{l}$ of diluted semen onto a slide and dragging the drop across the slide to cover an area of approximately $2.5 \mathrm{~cm} \times 5.0 \mathrm{~cm}$. Slides were air dried and fixed for $10 \mathrm{~min}$ in absolute ethanol.

Three protocols were used to stain the smears: (1) Rapid Diff II (Raymond A. Lamb, London), used according to the kit instructions; (2) Papanicolaou method (Sigma Diagnostics, Poole, Dorset), used in accordance with the instructions provided; and (3) Mayer's haematoxylin solution, for $20 \mathrm{~min}$ (Sigma Diagnostics) and alcoholic eosin $Y$ solution, for $3 \mathrm{~min}$ (Sigma Diagnostics). After staining, all slides were mounted with dibutyl phthalate xylene (DPX; BDH Laboratory Supplies, Poole, Dorset).

One ejaculate was subsequently collected from a purebred Meishan boar (Roslin Institute, Edinburgh) for comparison with the initial set of data. This ejaculate was collected, processed and assessed exactly as described for the other semen samples. The spermatozoa were stained with haematoxylin and eosin only.

MitoTracker midpiece staining. One ejaculate was subsequently collected from each of the three boars to investigate whether sperm tail length, as assessed by the HM package, was accurate. Live spermatozoa were stained with the mitochondria-specific probe MitoTracker Green FM (Molecular Probes Europe, Leiden) at a concentration of $100 \mathrm{nmol} 1^{-1}$, in accordance with the instructions provided. After staining, the spermatozoa were mounted with an antifade material (Citiflour; Agar, Stansted) and examined under an epifluorescent microscope $(\times 100$ objective). Spermatozoa were photographed and midpiece and tail length was measured using an eyepiece graticule. One thousand spermatozoa were assessed from each ejaculate.

\section{Morphology analysis}

Equipment. The HM computer-aided sperm analysis system (Hobson Tracking Systems Ltd, Sheffield) was used for the morphometric assessments. Slides were examined using an Olympus BH-2 microscope equipped with a brightfield $\times 40$ objective (numerical aperture 0.70 ) and a Sony SPT-M108CE monochrome video camera (Sony Corporation, Tokyo), linked via a X3.3 projection eyepiece. The video camera was equipped with automatic gain control (AGC) to ensure that the intensity of the image transferred to the computer was consistent. A green filter (IF 550, Olympus, Tokyo) was used to enhance image contrast and the illumination was set at a constant intensity.

Set-up procedure. Image recognition was optimized by setting a low numerical aperture on the microscope. This was achieved by opening the condenser iris diaphragm to the point where the defraction halo is about to disappear. This occurs at a condenser numerical aperture of 0.85 . A slide micrometer was used to calibrate the system. The number of spermatozoa analysed in one file was 200.

After the capture of an image, the system created an image Downloaded from Bioscientifica.com at 04/26/2023 10:23:41AM via free access 
histogram which displayed the brightness of individual pixels across the image analysis window in relation to their frequency. A histogram equalization process was carried out by assessing the image histogram, limiting the range of brightness used and allowing the contrast of the image to be improved. A low contrast image results in reduced image recognition and incorrect morphology analysis. Manual adjustment of the image histogram to occupy the length of its $x$-axis alters the histogram equalization settings used for image processing, allowing the contrast of the image to be further improved. The histogram was adjusted to occupy the entire $x$-axis to optimize the set-up of the image processor for recognition of boar spermatozoa. The HM produced an outline of blue dots around the captured sperm heads to allow the operator to check set-up parameters against image recognition. The HM also traced the tail of the spermatozoa, allowing the technician to check that the entire length was recognized. When the system was set-up for boar sperm as described, the dots closely surrounded the head without crossing the acrosome and the tail was correctly traced.

Initially, a random field on each slide was chosen for microscopic analysis. Subsequent analysis was performed on adjacent fields until 200 spermatozoa were counted. Before assessment by the HM package, each microscope field was examined manually to ensure that the head and entire length of the sperm tail were in the plane of focus. Images of spermatozoa from each field were captured, digitized on the basis of image pixel intensity and converted into a graphic image from which the processing software measured morphology parameters. All measurements were made after histogram equalization. Morphology measurements were obtained for the length of sperm tails $(\mu \mathrm{m})$, and the width $(\mu \mathrm{m})$ and length of sperm heads $(\mu \mathrm{m})$. The precision of each measurement was $0.1 \mu \mathrm{m}$. The HM package stores all the images assessed digitally. All stored images were examined visually to ensure that incorrect measurements were not made.

\section{Statistical analysis}

Summary statistics for the morphology data from each boar, ejaculate and slide were calculated. The Shapiro Wilk's $W$ test was applied to assess statistical 'normality'. Logtransformed values were subjected to one way analysis of variance (ANOVA) to examine random and systematic sources of variation. Specific contrasts were explored further using orthogonal coefficients within the ANOVA. Betweenslide effects were assessed by calculation of coefficients of variation.

\section{Multivariate analysis}

A multivariate pattern analysis was carried out using the PATN package (CSIRO, Canberra) to identify naturally occurring subgroups within the data set. Untransformed data were used in the multivariate analysis.

The multivariate analysis consisted of a number of sequential stages. First, the spermatozoa were placed in groups according to their morphometric measurements, producing a matrix of associations within the data. This nonhierarchical classification is carried out using the allocation (ALOC Module) algorithm. The number of resultant groups is dependent upon the value of the Bray and Curtis association index, selected at the start of each analysis. In the present study, the threshold association index was set at 0.3 , which produced six initial sperm subgroups.

The six subgroups were expressed mathematically as centroids and progressively joined into fewer groups by a second PATN algorithm, the 'sequential agglomerative hierarchical combinatorial flexible unweighted pair groups median average' (SAHN UPGMA) to process this information further. SAHN UPGMA scanned the six groups to locate the closest pair of values; these values were grouped together, and a new association between this group and all other subgroups was calculated. This process was repeated until there was only one group.

This fusion process can be represented graphically by producing a dendrogram showing the scheme of data linkage. Centroids that are close in the dendrogram are similar. Conversely, those separated by large distances are dissimilar in terms of the morphologies they represent. The number of sperm subgroups is reduced by cutting the dendrogram at an appropriate point on the association distance axis recommended by the PATN FUSE module.

The morphology characteristics of individual spermatozoa represented by the original six group centroids are used to calculate new characteristics describing the newly formed sub-groups. The principal co-ordinates analysis (PCoA) module is used next to reduce the number of morphology parameters needed to identify the subgroups of spermatozoa. A multiple linear regression programme, principal axis correlation (PCC), assesses how well the reduced number of morphology variables, defined by the PCoA, describes the original sperm groupings on the basis of all morphological parameters.

\section{Hypothesis testing}

After the pattern analysis, subpopulations determined by sperm morphology parameters were identified in each ejaculate. The untransformed, relative frequencies of spermatozoa from individual boars, in each subpopulation, were examined by chi-squared test.

More detailed information on the statistical modules and procedures used by PATN can be found in the programme manual (Belbin, 1993) and in texts on multivariate statistical analysis (Romesburg, 1984).

Statistical analyses were performed using Statistica for Windows, version 4.5 (Statsoft UK, Letchworth).

\section{Results}

The intensity of staining produced with the Rapid Diff II and Papanicolaou methods was insufficient to allow recognition and evaluation of spermatozoa, despite attempts to improve the contrast of the image by optimizing microscopic (low 
Table 1. Means $\pm S D$ of boar sperm morphology measurements from the entire data set

\begin{tabular}{lcc}
\hline Parameter & Number of spermatozoa & Mean \pm SD $(\mu \mathrm{m})$ \\
\hline Head length & 15000 & $8.60 \pm 1.09$ \\
Head width & 15000 & $4.77 \pm 0.78$ \\
Tail length & 15000 & $46.38 \pm 26.24$ \\
\hline
\end{tabular}

numerical aperture, green filter, AGC) and computer set-up procedures (histogram equalization). Therefore, all morphology measurements reported here were made on slides stained with haematoxylin and eosin, which produced consistent, strong staining in all areas of the sperm head and tail.

\section{Influence of boar on sperm morphology assessment}

Mean $\pm S D$ values for morphometric parameters taken from the entire data set are shown (Table 1). The frequency distributions of sperm morphometric values for tail length, head width and head length from the whole data set, are shown (Fig. 1). As these parameters were not normally distributed $(P<0.0001)$, subsequent ANOVA calculations were carried out on log-transformed values, which normalized the data.

The data for the three boars are summarized in a series of box whisker plots (Fig. 2). The ANOVA was calculated to investigate differences among the three boars, using the inter-ejaculate mean square as an error term for the boareffect $F$ value. A significant difference in variation among boars was observed for tail length $(P=0.001)$, but not for the width of the sperm head $(P=0.114)$ (Table 2$)$. The length of the head is reduced in the Sireline Large White boar, although this is not significant $(P=0.069)$ (Table 2). The mean values for tail length in all boars are shown (Table 3 ).

\section{Influence of ejaculate on sperm morphology assessment}

No significant difference among ejaculates was found for tail length $(P=0.256)$ and head length $(P=0.183)$ (Table 2$)$. However, a significant $(P=0.037)$ ejaculate effect was observed for the width of the sperm head (Table 2). Further exploration of the data revealed no significant difference $(P>0.938)$ among sperm head widths from ejaculates $1-4$, but ejaculate 5 in all boars had significantly smaller $(P<0.004)$ sperm head widths compared with the other ejaculates (Fig. 3).

\section{Influence of slide preparation on sperm morphology assessment}

No significant difference among slides was detected for measurements of sperm tail length $(P=0.770)$, head width $(P=0.736)$ and head length $(P=0.615)$ (Table 2$)$, indicating that both the staining and morphological analysis were precise and reproducible. Examination of coefficients of variation (CV) among slides, within boars, showed that

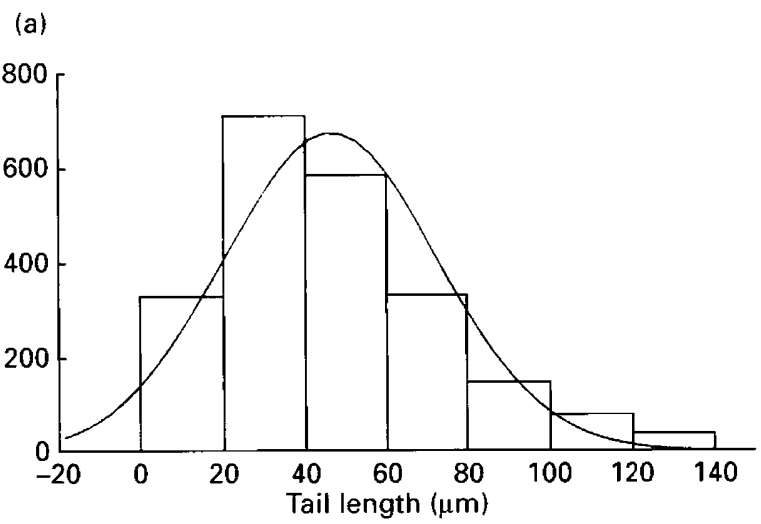

(b)

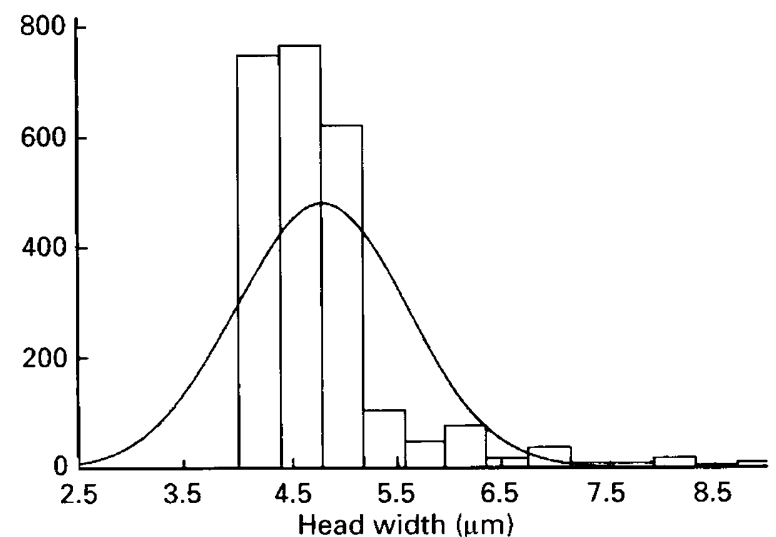

(c)

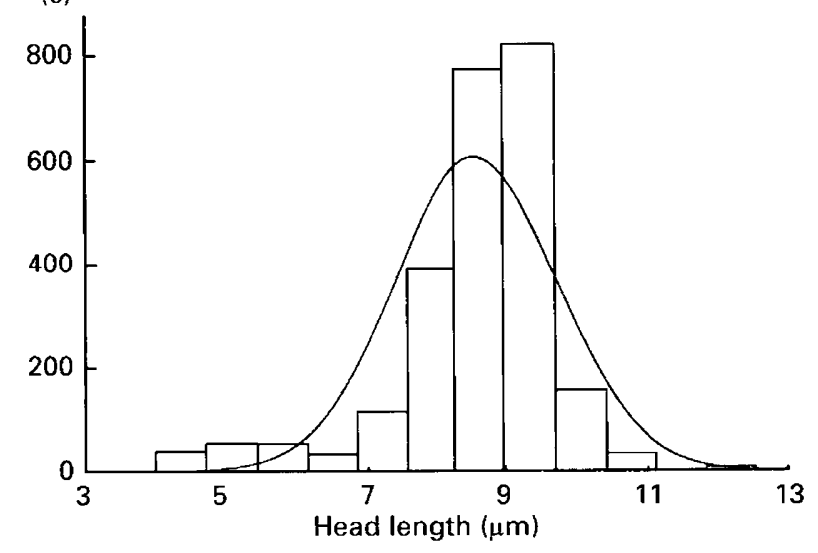

Fig. 1. Frequency distributions showing sperm morphometric values for (a) tail length, (b) head width and (c) head length obtained from the whole data set. Curve shows the expected normal distribution for this data.

precision was excellent for measurements of head length (Landrace-Meishan, 1.69\%; Sireline Large White, 2.18\%; Damline Large White, $2.31 \%$ ) and head width (LandraceMeishan, 3.08\%; Sireline Large White, $1.81 \%$; Damline Large White, $2.82 \%$ ) although CVs were higher for tail length (Landrace-Meishan, 29.04\%; Sireline Large White, $17.71 \%$; Damline Large White, $17.50 \%$ ). The tail length CV was higher for the Landrace-Meishan introgression boar, as was the actual tail length (Fig. 2a). The CV for the LandraceMeishan boar was influenced by the presence of a large percentage of spermatozoa from the subpopulation with 

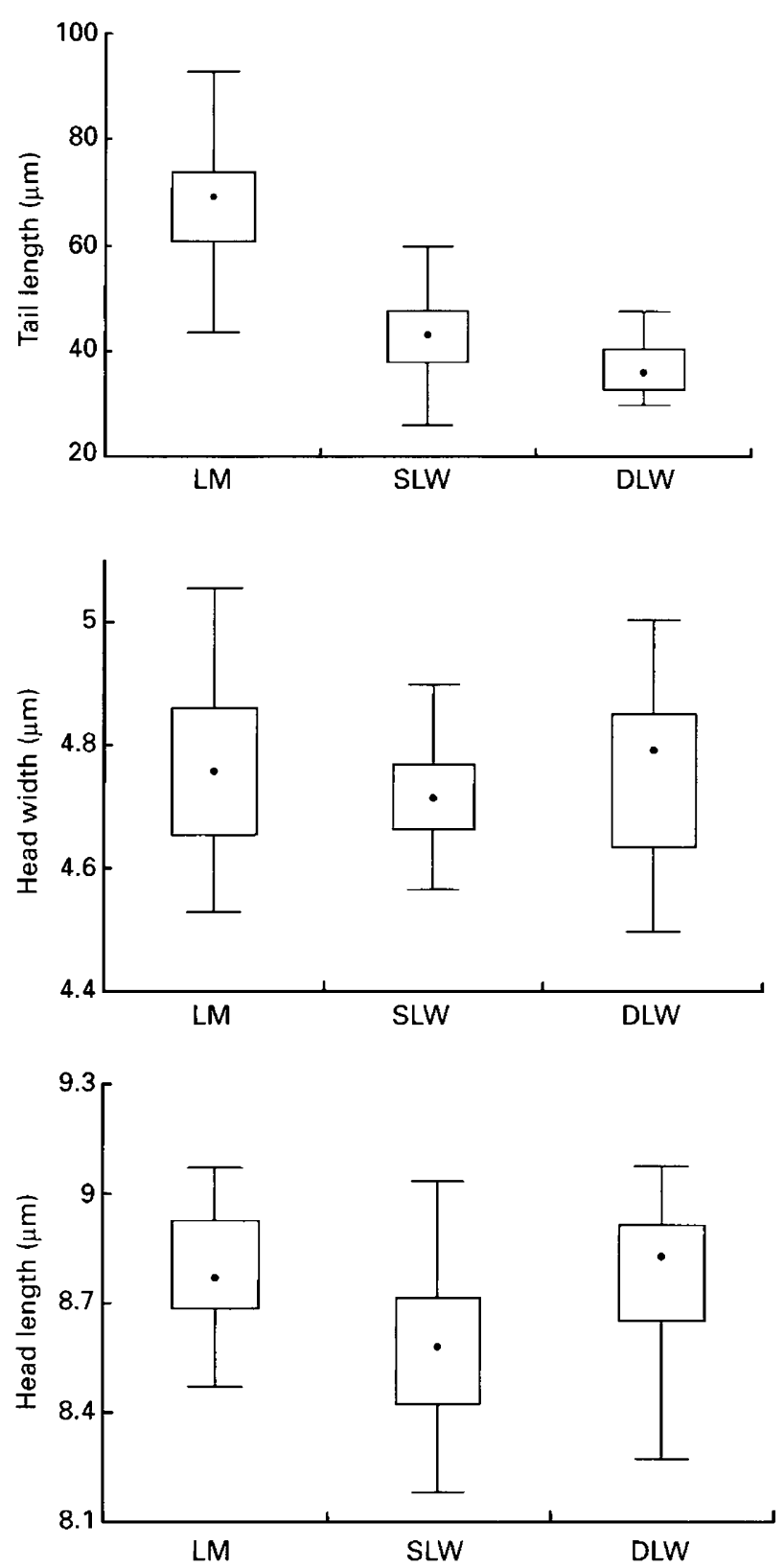

Fig. 2. Box whisker plots showing sperm morphometric values $(\mu \mathrm{m})$ for each of three boars: Landrace-Meishan introgression (LM), Sireline Large White (SLW) and Damline Large White (DLW). Boxes ( $\square$ ) enclose the 25 th and 75 th percentiles, $O$ is the median. Whiskers extend to the 5 th and 95 th percentiles.

long tails (49.2\%) compared with the Sireline $(29.9 \%)$ and Damline Large White (20.9\%) boars (Table 4 ).

\section{PATN analysis of sperm morphology measurements}

The boar sperm data analysed by PATN consisted of 3000 individual spermatozoa (observations) and three variables (tail length, head width and head length). One thousand spermatozoa were analysed from each of the three boars.

Six group centroids were produced by the ALOC algorithm from a non-hierarchical classification of the sperm measurements. The number of group centroids was reduced to three by cutting the dendrogram at an appropriate point on the association distance axis recommended by the SAHN UPGMA programme (Fig. 4). Three sperm groups were identified when the dendrogram was cut at any point between 0.33 and 0.46 on the association distance axis.

The three distinct groups of spermatozoa identified by PATN were defined using all three morphology variables; head length, head width and tail length. Although all variables were used in the analysis, ultimately, it was tail length that had most influence on the definition of subpopulations (Fig. 5). Head length and width had little influence when assigning the spermatozoa to a group. The three subpopulations consisted of spermatozoa with tail lengths ranging from 10.0 to $22.0 \mu \mathrm{m}$ in group 1, from 22.1 to $73.0 \mu \mathrm{m}$ in group 2 and from 73.1 to $130.0 \mu \mathrm{m}$ in group 3 (Fig. 6).

The frequencies of spermatozoa from each boar, allocated to the three sub-groups, were compared to see whether any of the groups contained proportionately more spermatozoa from a specific animal (Table 4). It was hypothesized that, if the large range of tail length measurements was caused by technical artefacts rather than biological variation, then all boars should be represented equally in each group. There was no significant difference in the distribution of spermatozoa from the three boars in group $1(P=0.230)$ and group $2(P=0.215)$. However, there were significantly more spermatozoa from the Landrace-Meishan introgression boar in group $3(P<0.0001)$. This finding supports the view that the Landrace-Meishan boar produced more spermatozoa with exceptionally long tails and that this observation is not an artefact. The hypothesis that spermatozoa with long tails were present in the ejaculate was further supported by the larger mean values for tail length in the Landrace-Meishan boar (Table 3) compared with the Sireline and Damline Large White boars. The spermatozoa with long tails were later identified using light microscopy and photographed (Fig. 6).

MitoTracker stain was used to investigate further whether the three subpopulations consisted of spermatozoa with intact tails or if the population with tails $10.0-22.0 \mu \mathrm{m}$ was made up of damaged spermatozoa. The MitoTracker Green FM accumulates in the active mitochondria of live spermatozoa with intact tails, producing a green fluorescence. Spermatozoa with broken tails were MitoTracker negative. Mitochondrial staining was observed in the midpieces of spermatozoa in each of the subpopulations (Fig. 7), confirming that short tails were indeed intact. Midpiece length was also examined to investigate whether short-tailed spermatozoa had a shorter midpiece. There were no significant differences in the length of the midpieces among the three subpopulations of spermatozoa $(P=0.992)$. The mean midpiece lengths and standard deviations were $12.20 \pm$ $1.20 \mu \mathrm{m}$ for the Sireline Large White boar, $12.30 \pm 1.10 \mu \mathrm{m}$ for the Damline Large White boar, and $12.20 \pm 1.00 \mu \mathrm{m}$ for the Landrace-Meishan boar.

\section{Meishan boar morphology}

Morphometric parameters for sperm tail length, head length and head width were assessed in a subsequent study 
Table 2. ANOVA for sources of variation in sperm dimension measurements

\begin{tabular}{|c|c|c|c|c|c|c|c|}
\hline Parameter & Source & $d f$ & $S S$ & MS & $E T$ & $F^{\mathrm{a}}$ & $P$ \\
\hline \multirow[t]{4}{*}{ Tail length } & Boar (B) & 2 & 4.0599 & 2.0300 & 0.0408 & 49.754 & $0.001^{*}$ \\
\hline & Ejaculate (E) & 4 & 0.1632 & 0.0408 & 0.0202 & 2.020 & 0.256 \\
\hline & Slide (S) & 4 & 0.0808 & 0.0202 & 0.0363 & 0.453 & 0.770 \\
\hline & $\mathrm{B} \times \mathrm{E} \times \mathrm{S}$ & 32 & 1.1616 & 0.0363 & & & \\
\hline \multirow[t]{4}{*}{ Head width } & Boar & 2 & 0.0018 & 0.0009 & 0.0023 & 3.913 & 0.114 \\
\hline & Ejaculate & 4 & 0.0092 & 0.0023 & 0.0003 & 7.667 & $0.037^{*}$ \\
\hline & Slide & 4 & 0.0012 & 0.0003 & 0.0006 & 0.500 & 0.736 \\
\hline & $\mathrm{B} \times \mathrm{E} \times \mathrm{S}$ & 32 & 0.0192 & 0.0006 & & & \\
\hline \multirow[t]{4}{*}{ Head length } & Boar & 2 & 0.0090 & 0.0045 & 0.0008 & 5.625 & 0.069 \\
\hline & Ejaculate & 4 & 0.0032 & 0.0008 & 0.0003 & 2.667 & 0.183 \\
\hline & Slide & 4 & 0.0012 & 0.0003 & 0.0005 & 0.667 & 0.615 \\
\hline & $\mathrm{B} \times \mathrm{E} \times \mathrm{S}$ & 32 & 0.0160 & 0.0005 & & & \\
\hline
\end{tabular}

aANOVA was calculated using the inter-ejaculate means square (MS) as an error term (ET) for the boar effect $F$ value, the inter-slide $M S$ as the $E T$ for the ejaculate effect and the MS of all remaining interactions as the ET for slide effects. Data were log transformed. $d f$, degrees of freedom; SS, sum of squares. *Significant.

Table 3. Means \pm sd of sperm tail length measurements for each of the four boars used in the present study

\begin{tabular}{ll}
\hline Boar & Tail length $($ mean $\pm \mathrm{SD})$ \\
\hline Landrace-Meishan introgression & $67.90 \pm 21.80$ \\
Sireline Large White & $43.66 \pm 7.71$ \\
Damline Large White & $40.47 \pm 11.72$ \\
Meishan & $66.36 \pm 24.70$ \\
\hline
\end{tabular}

of an individual Meishan boar. This additional evaluation was undertaken to investigate the likelihood that the long sperm tails identified in the Landrace-Meishan boar could also be detected in a pure breed Meishan boar. Mean head length (mean \pm SD: $8.66 \pm 1.30 \mu \mathrm{m}$ ) and width (mean \pm SD: 4.7 $\pm 0.60 \mu \mathrm{m}$ ) were assessed in Meishan spermatozoa. Six per cent of the total number of spermatozoa had tails 10.0-22.0 $\mu \mathrm{m}, 70 \%$ had tails $22.1-73.0 \mu \mathrm{m}$ and $24 \%$ had tails $73.1-130.0$ $\mu \mathrm{m}$ in length. The presence of exceptionally long sperm tails was confirmed in this ejaculate in which $24 \%$ of the tails were in the range $73.1-130.0 \mu \mathrm{m}$.

\section{Discussion}

Ideally, sperm morphology analysis should be accurate, producing precise and reproducible measurements. The current evaluation techniques for morphology rely on a highly subjective visual assessment, involving the classification by light microscopy of normal spermatozoa from stained smears, which makes morphology parameters difficult to quantify (Davis and Gravance, 1993; Davis et al., 1995). Indeed, the definition of a 'normal' spermatozoon is, in itself, subjective and contributes little to the study and classification of sperm subpopulations that may possess morphological attributes outside the 'normal' criteria. The introduction of ASMA has allowed rapid, accurate and

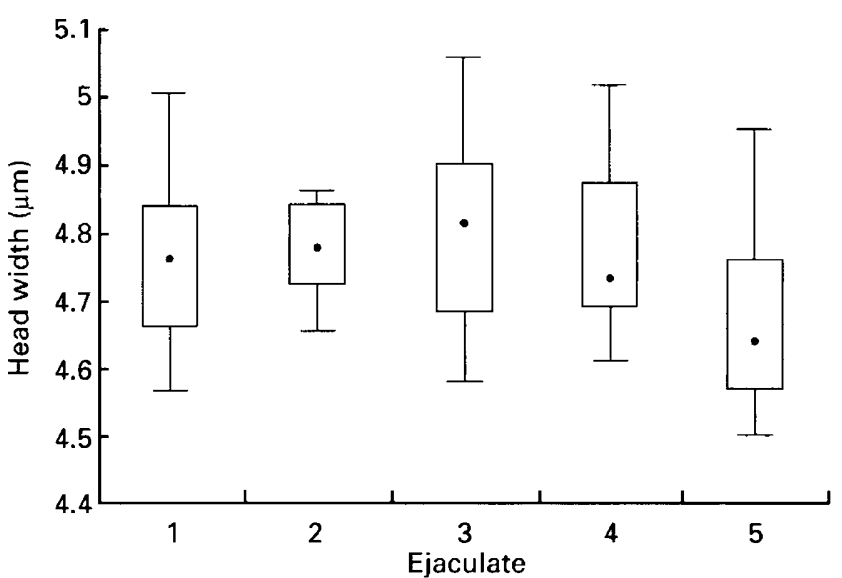

Fig. 3. Box whisker plot showing sperm head width measurements $(\mu \mathrm{m})$, for each of the five ejaculates from all five boars. Boxes $(\square)$ enclose the $25^{\text {th }}$ and $75^{\text {th }}$ percentiles, is the median. Whiskers extend to the 5 th and 95 th percentiles.

reproducible evaluation, providing an objective basis from which to study sperm morphology (Perez-Sanchez et al., 1994; Ball and Mohamed, 1995; Comhaire and Vermeulen, 1995; Gravance and Davis, 1995; Kruger et al., 1996; Dahlbom et al., 1997). It is now simple to collect a large data set composed of thousands of individual sperm parameters in a relatively short time. The present study was based on a data set of 15000 spermatozoa. Previous studies have used ASMA systems for morphological classification in a wide range of species, including humans (Perez-Sanchez et al., 1994), goats (Gravance et al., 1995) and dogs (Dahlbom et al., 1997). However, little information is available for boar spermatozoa.

The accuracy of sperm measurements made by the ASMA equipment depends upon a number of analytical variables: namely preparation, fixation and staining of the spermatozoa (Davis and Gravance, 1993; Ombelet et al., 1995; Gago et al., 1998). The cellular components of spermatozoa, across 
Table 4. Variation in the proportion (\%) of spermatozoa that make up subgroups 1, 2, and 3 from each of the three boars used in the present study

\begin{tabular}{lcccrr}
\hline Group & $\begin{array}{c}\text { Landrace-Meishan } \\
\text { introgression }\end{array}$ & Sireline Large White & Damline Large White & Total & Chi-squared \\
\hline 1 & 30.5 & 36.7 & 32.8 & 100 & 2.938 \\
2 & 32.3 & 34.4 & 33.3 & 100 & 0.2302 \\
3 & 49.2 & 29.9 & 20.9 & 100 & 3.076 \\
\hline
\end{tabular}

Hypothesis: If long tail morphology is an error reading by the Hobson morphology package, each boar should have the same number of errors. If the long tail subgroup is composed of more spermatozoa from a specific boar, the presence of long tails may be a correct observation. Figures represent the proportion (\%) of spermatozoa which make up subgroups 1-3 from the three boars. Group 1: spermatozoa with tail lengths 10.0-22.0 $\mu \mathrm{m}$; group 2: 22.1-73.0 $\mu \mathrm{m}$; and group 3: 73.1-130.0 um. *Signficant.

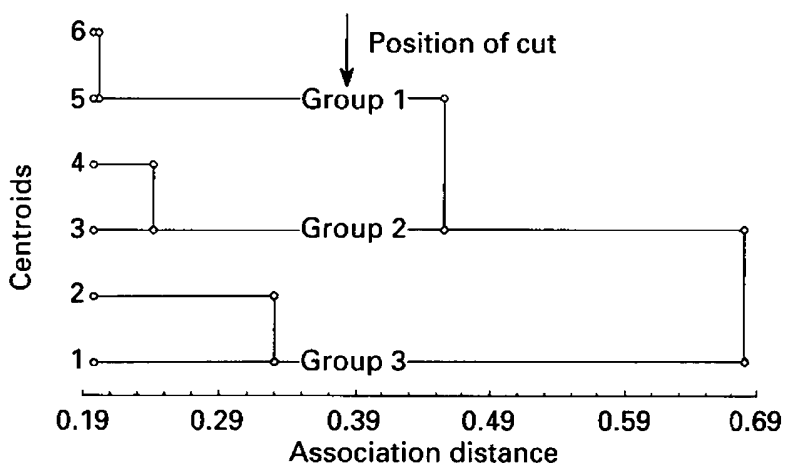

Fig. 4. Dendrogram derived by PATN computer package from boar sperm data. Numbers on the left of the plot represent centroids formed by fusion of individual sperm morphology values. Three populations of spermatozoa were defined by cutting the dendrogram at the position shown along the association distance axis.

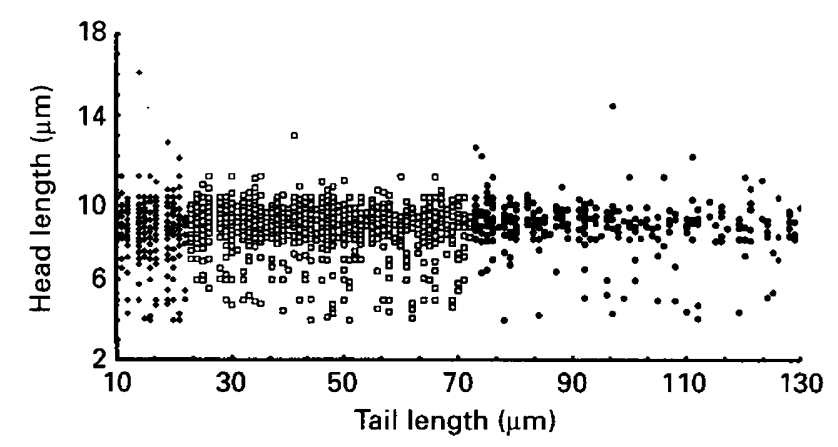

Fig. 5. Scattergram showing sperm tail and head length measurements in relation to PATN computer package-derived classifications. Each point represents an individual spermatozoon. Group 1: (10.0-22.0 $\mu \mathrm{m})$; group 2: $\square(22.1-73.0 \mu \mathrm{m})$; and group 3: (73.1-130.0 $\mu \mathrm{m})$.

species, react differently with dyes and fixatives from different preparation methods (Harasymowycz et al., 1976). Therefore, it is imperative that the best preparation and staining protocols are chosen for use in a particular species, producing precise and repeatable morphology evaluation. This would enable techniques to be applied in different laboratories with consistent results, thus, introducing a degree of quality control into ASMA technology.

The Rapid Diff II staining method was investigated after
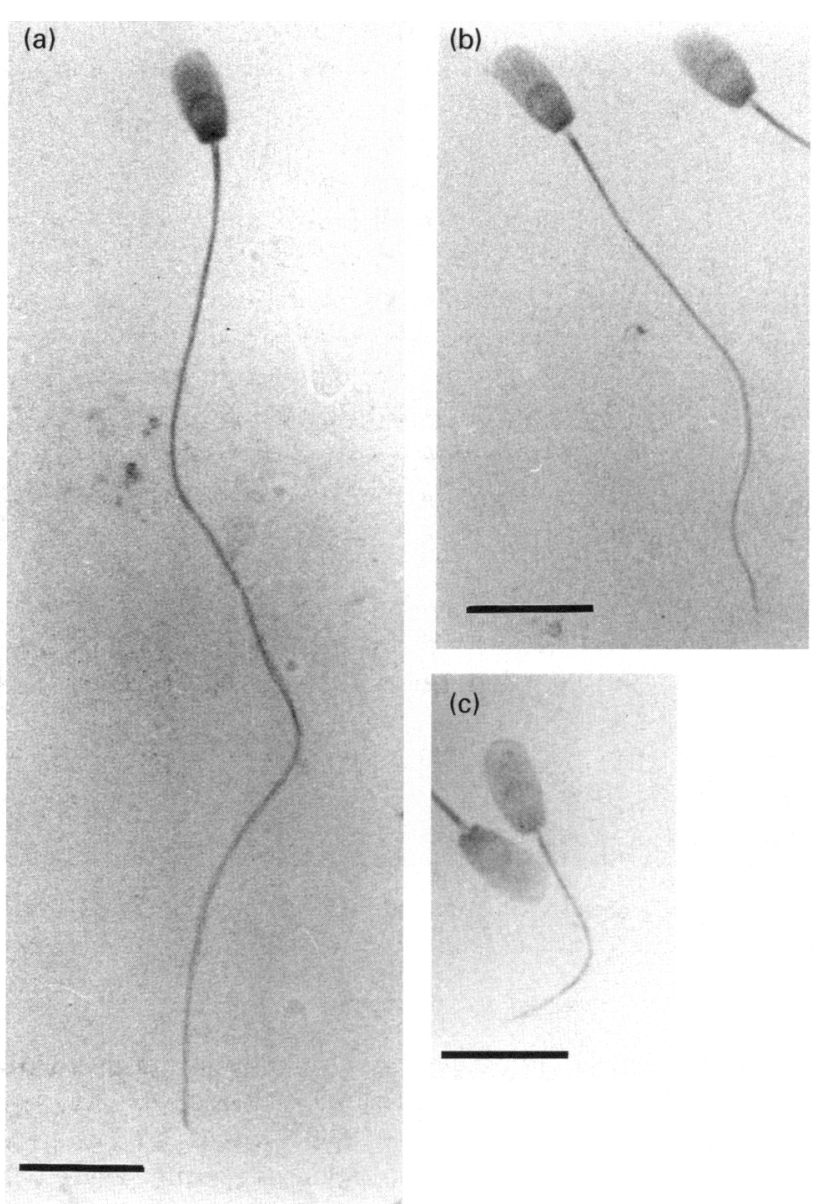

Fig. 6. Examples of spermatozoa from the three subpopulations identified by PATN computer package with tail lengths: (a) $87 \mu \mathrm{m}$ (group 3); (b) $43 \mu \mathrm{m}$ (group 2); and (c) $19 \mu \mathrm{m}$ (group 1). Scale bars represent $10 \mu \mathrm{m}$.

its use to establish strict criteria for human spermatozoa (Kruger et al., 1993), and both Papanicolaou and haematoxylin and eosin have proven useful with spermatozoa from nonhuman species (Gravance et al., 1995; Perez-Sanchez et al., 1997). In the present study, the intensity of spermatozoa staining with Rapid Diff II and Papanicolaou was insufficient for recognition by the HM. However, haematoxylin and eosin staining produced high contrast between the spermatozoa and the background, allowing good definition of the sperm 

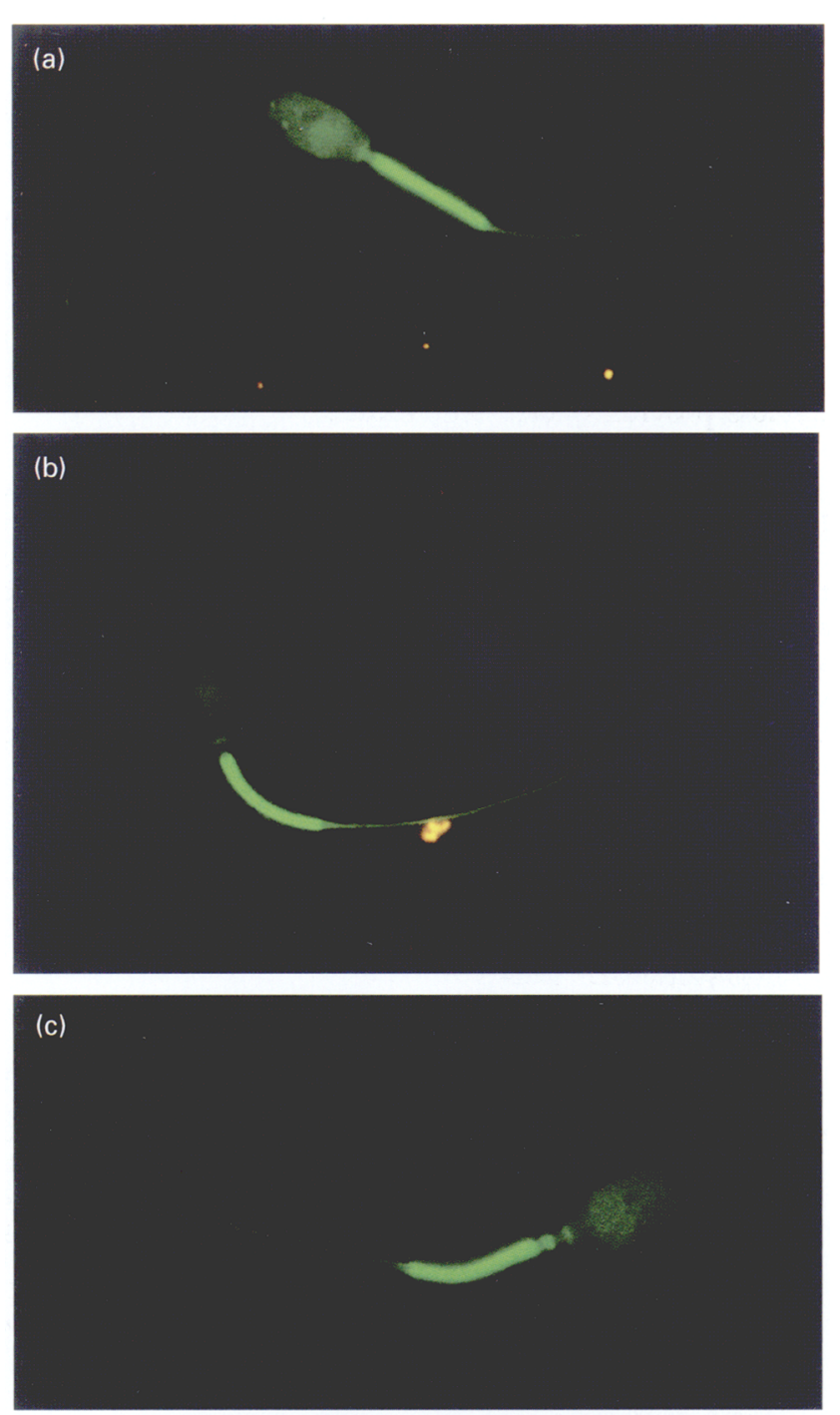

Fig. 7. Live spermatozoa from the three subpopulations identified by PATN computer package-stained with MitoTracker Green FM probe.

boundary. Careful adjustment of the iris diaphragm helped to define the cell boundaries even more clearly.

The dilution stage used in the present study is not necessary for standard morphology assessments made with the package, although it is advisable to smear at a concentration of $100 \times 10^{6} \mathrm{ml}^{-1}$, or less, to prevent the formation of clusters of spermatozoa on the slide. This clustering disrupts recognition of sperm by the HM. Smears made from spermatozoa at a concentration of $100 \times 10^{6} \mathrm{ml}^{-1}$ were assessed easily by the morphology package.

The suitability of the specimen preparation method was evaluated by analysing the variation among smears. No significant differences among slides were detected, indicating that both the staining and morphology analysis were precise and reproducible. The coefficient of variation among slides was very low for measurements of sperm head length and width; tail length measurements were somewhat higher. This finding indicates that the HM is an accurate means of measuring all morphology parameters.
Coefficients of variation were higher for tail measurements, as lengths were more variable.

The variability in head width measurements among ejaculates was surprising. Further investigation revealed that ejaculate number 5 , in all boars, exhibited significantly lower values for head width. It was established that these ejaculates were collected and prepared as a batch on the same day. From this information, it was concluded that a new variable, such as a change in temperature, osmolarity of diluents or handling of the sample, was introduced accidentally into the experiment during collection, transport or preparation of ejaculate 5, causing changes to the spermatozoa. An interesting outcome of this observation was that sperm head and tail lengths were unaffected by this new variable, indicating that head width is a more sensitive morphology parameter and may be used to indicate damage to the spermatozoa. In addition, the small magnitude of the difference in head width detected $(0.1 \mu \mathrm{m})$ demonstrates the sensitivity of the HM system.

The mean values obtained in this study for morphology parameters from the entire data set are in broad agreement with previous pig sperm dimensions reported by Cummins and Woodall (1985). However, exploration of the data revealed a large SD for tail length, indicating a wide range of individual measurements. Closer inspection of the data was undertaken to establish the source of variation.

The large data sets obtained from ASMA are best investigated using a multivariate approach rather than more conventional variance analyses that assume, often incorrectly, a normal distribution of morphology measurements.

The entire morphology data set, assimilated during this investigation, was examined by a multivariate pattern analysis to explore the heterogeneity of the morphology measurements and identify the presence of subpopulations. The PATN method of data analysis (Belbin, 1993) has been applied successfully to define subpopulations of spermatozoa on the basis of motion characteristics (Abaigar et al., 1999). Three subpopulations of spermatozoa were identified and defined by tail lengths: 10.0-22.0, 22.1-73.0 and $73.1-130.0 \mu \mathrm{m}$. The lack of variation in sperm head length and width among boars is in agreement with the claim that these represent genuinely distinct subpopulations that may differ functionally. Initially, it was thought that the subpopulation with tail lengths of 10-22.0 $\mu \mathrm{m}$ may consist of spermatozoa with broken tails; however, the absence of any unattached tails in the smears does not support this view.

MitoTracker, which was used to investigate flagellar integrity in more detail, accumulated in the active mitochondria of live spermatozoa with intact tails, producing green fluorescence. Spermatozoa with broken tails had no mitochondrial activity and were MitoTracker negative. Mitochondrial staining of midpieces was observed in spermatozoa in each of the subpopulations, confirming that short tails were indeed intact. Midpiece length was also examined to investigate whether short tailed spermatozoa had shorter midpieces. Spermatozoa from the three subpopulations showed no significant differences in midpiece length. This result is notable since the midpiece contains the mitochondria and, therefore, its length may influence the amount of energy available to the spermatozoa. 
This finding indicates that spermatozoa with long and short tails have similar amounts of mitochondrial energy available for motility.

In the present study three subpopulations of spermatozoa were identified that exhibit variation in tail length, and the proportion of each subpopulation in an ejaculate was shown to vary among boars. However, the results cannot be interpreted in terms of breed differences, as the limited number of boars studied are not necessarily representative of their respective genetic lines. Nor can we conclude that all boar ejaculates will contain spermatozoa from these three subpopulations.

The evidence that there are morphologically distinct subpopulations of spermatozoa within the ejaculate has implications for more advanced ASMA systems developed to use a neuronal network that can be trained to recognize morphological classifications determined by the operator. Previous automated systems have been based solely on morphology assessments of sperm head characteristics. The current advanced systems allow automatic measurement of mid pieces and tails, as does the HM, supplementing the original morphometric data from the sperm head (Steigerwald and Krause, 1998). However, some caution must be applied to such measurements obtained from these systems. The training of a system to recognize morphological classifications introduces a subjective operator bias into an objective measurement protocol. The ASMA systems allow minimum and maximum values for morphology parameters of tail length and head length to be specified, rejecting measurements that lie outside these nominal values. A selection of 'normal' spermatozoa is created with the possibility of excluding a subpopulation beyond these parameters.

A number of morphologically distinct subpopulations of spermatozoa, distinguished by variation in tail length, were found within the boar ejaculate. The proportion of spermatozoa from each subpopulation present in the ejaculate varied among boars. In the present study, ejaculates from the Landrace-Meishan introgression boar contained significantly more spermatozoa from the subpopulation with long tails than did ejaculates from other boars. Subsequent analysis of spermatozoa from a pure-bred Meishan also showed the ejaculate to be composed of a significant number of spermatozoa from this subpopulation with tails longer than $73.0 \mu \mathrm{m}$. This observation is in agreement with the results from the Landrace-Meishan spermatozoa; however, further study is needed before it can be concluded that tail length is related to breed. Gomendio and Roldan (1991) showed that sperm tail length in mice is positively correlated with swimming speed, which implies that boars with a subpopulation of spermatozoa with long tails may be of high fertility. The Meishan breed is known to be highly fertile and the integration of its genetic characteristics into a second breed, producing the Landrace-Meishan introgression boar, is in agreement with the fertility hypothesis put forward by Gomendio and Roldan (1991). The suggestion that spermatozoa with longer tails are able to swim faster has implications for sperm competition theory in which subpopulations of spermatozoa could gain a competitive edge by progressing along the female tract faster than other spermatozoa. This evidence promotes considerable interest in the possibility that variability in subpopulation morphology reflects functional and adaptive differences and that the presence of specific subpopulations within ejaculates provides a selective fertility advantage.

A standard specimen preparation method and a number of ASMA package set-up parameters were developed for the analysis of porcine sperm morphology by the Hobson Morphology package. Using set-up parameters derived from the present study, the performance of the ASMA system was reliable, providing consistent results.

The authors are grateful to all staff at GTC South (Pig Improvement Company, Hungerford, UK) for their constant support and for supplying the boar semen used in this study. They also wish to thank G. Hobson and A. Mileham for many useful discussions and T. Abaigar for help with the statistics. This research was supported by grants from the BBSRC and the Pig Improvement Company, Oxford, UK.

\section{References}

Abaigar T, Holt WV, Harrison RAP and del Barrio G (1999) Sperm subpopulations in boar (Sus scrofa) and gazelle (Gazella dama mhorr) semen as revealed by pattern analysis of computer assisted motility assessments Biology of Reproduction $6032-41$

Ball BA and Mohamed HO (1995) Morphometry of stallion spermatozoa by computer assisted image analysis Theriogenology 49 367-377

Bedford JM (1983) Significance of the need for sperm capacitation before fertilisation Biology of Reproduction 28 108-120

Belbin L (1993) PATN, Pattern Analysis Package Division of Wildlife and Ecology, CSIRO, Canberra

Chang MC and Hunter RHF (1975) Capacitation of mammalian sperm: biological and experimental aspects. In Handbook of Physiology. Endocrinology $V$ pp 339-351 Eds DW Hamilton and RO Greep. American Physiological Society, Washington DC

Comhaire F and Vermeulen L (1995) Human semen analysis Human Reproduction Update 1343-362

Cummins JW and Woodall PF (1985) On mammalian sperm dimensions Journal of Reproduction and Fertility 75 153-175

Dahlbom M, Andersson M, Vierula M and Alanko M (1997) Morphometry of normal and teratozoospermic canine sperm heads using an image analyser: work in progress Theriogenology 48 687-698

Davis RO and Gravance CG (1993) Standardisation of specimen preparation, staining and sampling methods improves automated sperm head morphometry analysis Fertility and Sterility $59412-417$

Davis RO and Gravance CG (1994) Consistency of sperm morphology classification methods Journal of Andrology 15 83-91

Davis RO, Gravance CG, Thai DM and Miller MG (1995) Automated analysis of toxicant induced changes in rat sperm head morphometry Reproduction and Toxicology 8 521-529

Gago C, Perez-Sanchez F, Yeung CH, Tablado L, Cooper TG and Soler C (1998) Standardisation of sampling and staining methods for the morphometric evaluation of sperm heads in the Cynomolgus monkey (Macaca fascicularis) using computer assisted image analysis International Journal of Andrology 21 169-176

Gomendio M and Roldan ER (1991) Sperm competition influences sperm size in mammals Proceedings Royal Society of London B 243 181-185

Gravance CG and Davis RO (1995) Automated sperm morphometry analysis in the rabbit Journal of Andrology 16 88-93

Gravance CG, Lewis KM and Casey PJ (1995) Computer automated sperm head morphometry analysis (ASMA) of goat spermatozoa Theriogenology 44 989-1002

Harasymowycz J, Ball L and Seidel E (1976) Evaluation of bovine spermatozoal morphological features after staining or fixation American Journal of Veterinary Research 37 1053-1057

Harrison RAP (1996) Capacitation mechanisms and the role of capacitation as seen in eutherian mammals Reproduction Fertility and Development 8 581-594 
Jagoe JR, Washbrook NP, Pratsis L and Hudson EA (1987) Sperm morphology by image analysis compared with subjective assessments British Journal of Urology $60457-462$

Katz DF and Yanagimachi R (1980) Movement characteristics of hamster spermatozoa within the oviduct Biology of Reproduction 22 759-764

King GJ and Macpherson JW (1973) A comparison of two methods for boar semen collection Journal of Animal Science 36 563-565

Kruger TF, Du Toit TC, Franklin DR, Acosta AA, Oehninger SC, Menkveld $\mathbf{R}$ and Lombard CJ (1993) A new computerised method of reading sperm morphology (strict criteria) is as efficient as technician reading Fertility and Sterility 59 202-209

Kruger TF, Lacquet FA, Sanchez Sarmiento CA, Menkveld R, Ozgur K Lombard CJ and Franken DR (1996) A prospective study on the predictive value of normal sperm morphology as evaluated by computer (IVOS) Fertility and Sterility 66 285-291

Olds-Clarke P and Sego R (1992) Calcium alters capacitation and progressive motility of uterine spermatozoa from $+/+$ and congenic $t^{32 /+}$ mice Biology of Reproduction $47629-635$
Olds-Clarke P and Wivell $\mathbf{W}$ (1992) Impaired transport and fertilisation in vivo of calcium treated spermatozoa from $+/+$ or congenic $t^{32 / 4}$ mice Biology of Reproduction 47 621-628

Ombelet W, Menkveld R, Kruger TF and Steeno O (1995) Sperm morphology assessment: historical review in relation to fertility Human Reproduction Update 1543-557

Perez-Sanchez F, De Monserrat JJ and Soler C (1994) Morphometric analysis of human sperm morphology International Journal of Andrology 17 248-255

Perez-Sanchez F, Tablado L and Soler C (1997) Sperm morphological abnormalities appearing in the male rabbit reproductive tract Theriogenology 47 893-901

Roldan ERS, Cassinello J, Abaigar T and Gomendio M (1998) Inbreeding, fluctuating asymmetry and ejaculate quality in an endangered ungulate Proceedings Royal Society of London B 265 243-248

Romesburg H (1984) Cluster Analysis for Researchers Lifetime Learning publications, Belmont, CA

Steigerwald P and Krause W (1998) Estimation of sperm morphology using a new CASA system Andrologia 30 15-22 